\title{
XAS structural insights into Cu binding with amyloid Aß4-y peptides
}

Ruwini Supeshala Kumari Ekanayake ${ }^{1}$, Stephen Best ${ }^{2}$, Christopher Chantler ${ }^{1}$, Victor Streltsov ${ }^{3}$

${ }^{1}$ School Of Physics, The University Of Melbourne, Melbourne, Australia, ${ }^{2}$ School of Chemistry, The University of Melbourne, Melbourne, Australia, ${ }^{3}$ Florey Institute of Neuroscience and Mental Health, The University of Melbourne, Melbourne, Australia E-mail: rekanayake@student.unimelb.edu.au

Alzheimer's disease (AD) is a progressive neurodegenerative disorder characterized by the presence of amyloid (Aß) plaques. In addition to modulating senile $A B$ plaque formation, $A B-\mathrm{Cu}$ complexes are involved in extensive redox chemical reactions. The first protein sequencing studies of the $A \beta$ plaque core (APC) of $A D$ patients identified NH2-terminal heterogeneity and the majority $(64 \%)$ of the APC-AD AB peptides begins with a Phe4 residue. This $\mathrm{N}$-truncated sequence produced the highaffinity (dissociation Kd of femtoM) amino-terminal Cu Ni (ATCUN, H2N-Xaa-Yaa-His) binding motif.

X- ray absorption spectroscopy (XAS) combined with electrochemical control (XAS-SEC) was utilised to derive structural models for the interaction of CuI and CuII with N-truncated $A \beta$ peptides. First, the conventional XAS of CuII:AB1-16 and CuII:Aß4-y $(y=8,12,16)$ as frozen solutions $(5-10 \mathrm{~K}$, Fig. 1a,b) and secondly XAS-SEC at room temperature under potentiostatic control have been measured (Fig. 1c). The performance of the XAS-SEC cell in the Australian Synchrotron XAS beamline is demonstrated by the current response to a step potential and to application of a triangular waveform (Fig. 1d). The XAS of CuII:AB4 8 and CuII:Aß4-16 show the oscillations characteristic for CuII binding to the ATCUN site and prove that Tyr10 or Glu11 are NOT involved in CuII binding at pH of 7.4 in our studies. The binding geometry is different from the CuII binding site of Aß1-16 involving 3 Histidine: His6-His13-His14 (Fig. 1a) [1].

The reduction potential, $\mathrm{E}^{\circ}(\mathrm{CUII} / \mathrm{I})$, depends on the relative strength of CuII/CuI binding where the ATCUN motif available only for the truncated $A B 4-y$ peptide giving both higher $\mathrm{Kd}$ and more negative reduction potential, i.e. $E^{\circ}(\mathrm{CuII} / \mathrm{I}: \mathrm{A} 1 \mathrm{1}-16)>$ $E^{\circ}(C u I I / I: A ß 4-y)$. The reduction of CuII:Aß1-16 is irreversible with CuI rearrangement of the 3 His to almost linear geometry His13-His14 binding site [2]. While not observable by electrochemical measurements10, reduction of CuII:Aß4-y can be monitored by XAS-SEC. More forcing conditions are needed to reduce CuII:Aß4-12 than CuII:Aß4-16. CuI binding at the His13-His 14 site is inferred by the XAS analysis of spectra recorded during in situ reduction of CuII:AB4-16 (Fig. 1C).

These results suggest that reduction of CuII:Aß4-16 proceeds from CuII bound to the His13-His14 site (Aß416:CuIIHis13,14), possibly via His6-His14 "preorganization" [3] which is in equilibrium with AB4-16:CUIIATCUN.

[1] StreltsovV et al. Biophys, J 95 (2008) 3447-56

[2] Shin BK, Saxena S. J Phys Chem A, 115 (2011) 9590-9602; Furlan S et al. J Phys Chem B, 114 (2010) 15119-15133; Hureau C et al. J Biol Inorg Chem, 14 (2009) 995-1000; Himes RA et al. Angew Chem Int Ed Engl, 47 (2008) 9084-9087; Shearer J, Szalai VA. J Am Chem Soc, 130 (2008) 17826-17835.

[3] Balland V, Hureau C, Savéant J-M. Proc National Academy of Sciences 107 (2010) 17113-17118, Streltsov VA et al, manuscript in preparation

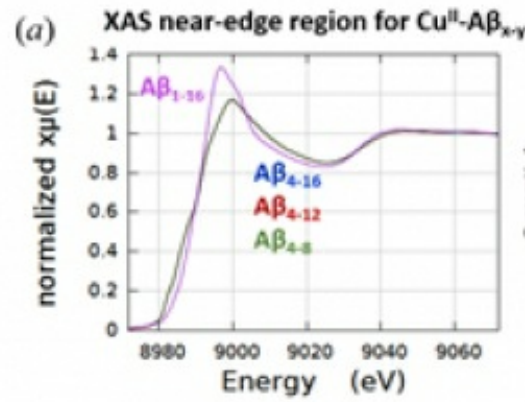

(b) XAS fit in $\mathrm{k}$-space for $\mathrm{Cu}^{\mathrm{n}}-\mathrm{A} \boldsymbol{\beta}_{4-\mathrm{\gamma}}(\mathrm{Y}=\mathbf{8}, \mathbf{1 6})$



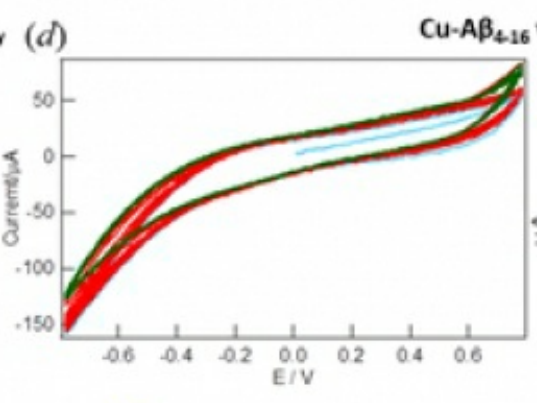

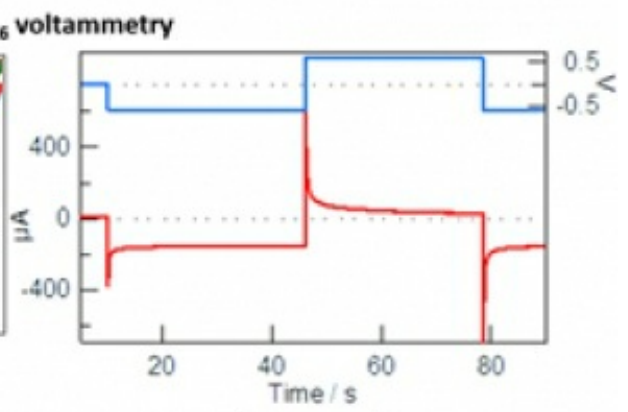

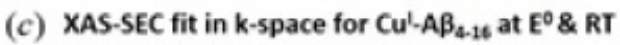

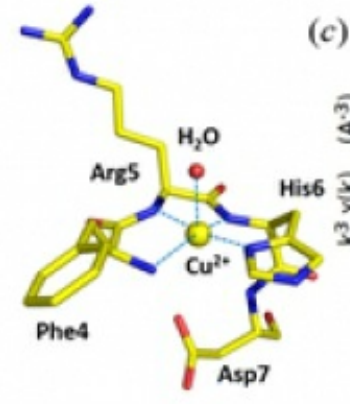



Wavenumber $\left(\mathrm{A}^{-1}\right)$

Keywords: XAFS, Alzheimer Disease, metal amyloid- $\beta$ complexes 